\title{
CLASSIFICATION OF BREAST CANCER MALIGNANCY USING CYTOLOGICAL IMAGES OF FINE NEEDLE ASPIRATION BIOPSIES
}

\author{
ŁUKASZ JELEŃ, Thomas FEVENS， ADAM KRZYŻAK \\ Department of Computer Science and Software Engineering \\ Concordia University \\ 1455 de Maisonneuve Blvd. West, Montréal, Québec, H3G 1M8, Canada \\ e-mail: $\left\{1 \_j e l e n\right.$, fevens, krzyzak\}@cse.concordia.ca
}

\begin{abstract}
According to the World Health Organization (WHO), breast cancer (BC) is one of the most deadly cancers diagnosed among middle-aged women. Precise diagnosis and prognosis are crucial to reduce the high death rate. In this paper we present a framework for automatic malignancy grading of fine needle aspiration biopsy tissue. The malignancy grade is one of the most important factors taken into consideration during the prediction of cancer behavior after the treatment. Our framework is based on a classification using Support Vector Machines (SVM). The SVMs presented here are able to assign a malignancy grade based on preextracted features with the accuracy up to $94.24 \%$. We also show that SVMs performed best out of four tested classifiers.
\end{abstract}

Keywords: Automated malignancy grading, FNA grading, SVM, breast cancer grading, Bloom-Richardson.

\section{Introduction}

Breast cancer is the most often diagnosed cancer among women aged 40 to 60 . According to the World Health Organization there are 7.6 million deaths worldwide due to cancer each year, out of which 502,000 are caused by breast cancer alone. With such a high rate, breast cancer also is one of the most deadly cancers. For many years researchers have been trying to find the best way to treat breast cancer. Successful treatment is a key to reduce the high death rate. To successfully cure a patient with breast cancer we need to diagnose it as early as possible. Cancers in their early stages are vulnerable to treatment while cancers in their most advanced stages are usually almost impossible to treat.

The most common diagnostic tools are mammography and a fine needle aspiration biopsy (FNA). Mammography, which is a non-invasive method, is most often used for screening purposes rather than for precise diagnosis. It allows a physician to find possible locations of microcalcifications and other indicators in breast tissue. When a suspicious region is found, the patient is sent to a pathologist for a more precise diagnosis. This is when the FNA is taken. A fine needle aspiration biopsy is an invasive method to extract a small sample of the questio- nable breast tissue that allows the pathologist to describe the type of the cancer in detail. Using this method pathologists can very adequately describe not only the type of the cancer but also its genealogy and malignancy. They can also foresee the course of cancer development by attributing a predictive factor to it. The stage of cancer depends on the malignancy factor that is assigned during an FNA examination. The determination of malignancy is essential when predicting the progression of cancer.

In the literature one can find approaches to breast cancer classification (Wolberg and Mangasarian, 1990; Wolberg, Street and Mangasarian, 1994; Street, 2000; Walker and Albertelli, 1998; Walker, Albertelli, Titkov, Kaltsatis and Seburyano, 1998; Nezafat, Tabesh, Akhavan, Lucas and Zia, 1998; Schnorrenberg, Pattichis, Kyriacou and Schizas, 1994). All of these approaches are concentrated on classifying FNA slides as benign or malignant. The system presented in this study classifies a malignancy stage of cancer that is nearly always malignant due to the prescreening process before taking an FNA.

During examination, there are certain features in the cytological slides that are taken into account. These features are used to assign a grade to the diagnosed tissue. One of the most popular and widely used schemes for 
grading cytological tissue is the Bloom-Richardson grading scheme (Bloom and Richardson, 1957). This system was originally introduced for grading breast cancer histological pictures. The same scale can easily be used to assess malignancy for cytological smears. According to this system, there are three factors that are taken into account while grading cancerous tissue. Each of the three factors is evaluated on a three-point scale according to the following description:

1. Degree of structural differentiation (SD): In histopathological slides this is also described as tubule formation, which reflects cell tendency to form tubules. Since in cytological smears tubules are not preserved, the following scoring for this factor is based on the classification of cell groupings within a smear, see, e.g., Fig. 1 .

- One point: cells in the image are grouped regularly.

- Two points: both grouped and single cells can be found within the image.

- Three points: cells are spread irregularly.

2. Pleomorphism (P): This factor takes into consideration differences in size, shape and staining of the nuclei. This scoring is fairly straightforward because with the growth of irregularity of the nuclei the prognosis becomes worse. Figure 2 shows an example of these variations. We have the following scoring:

- One point: nuclei with uniform size, shape and staining.

- Two points: moderate variations are found.

- Three points: very significant variations.

3. Frequency of hyperchromatic and mitotic figures (HMF): Mitosis is a process in the cell life cycle in which a mother cell is divided into two identical cells. Figure 3 shows an example of mitosis. In the center part one can notice a thin border between two nuclei. A darker shade of staining can also be seen. The main objective of this factor is to assess the number of mitotic figures in the viewed field. Several fields of view on the same slide are taken into account because this step is done in large magnification. The more cases of mitosis found, the worse the prognosis. We have the following scoring:

- One point: occasional figures per field are found.

- Two points: smears with two or three figures in most fields.

- Three points: more than three figures per field are found.

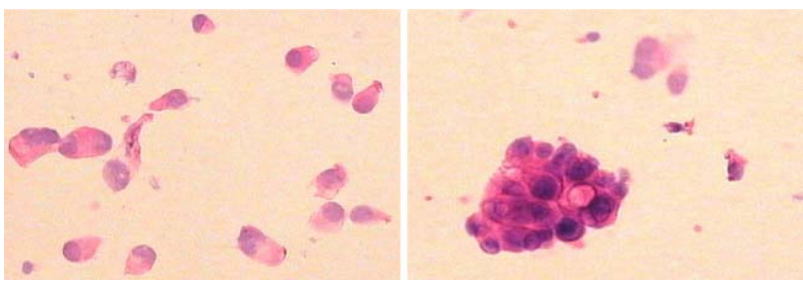

Fig. 1. Cell groupings: (a) spread cells, (b) grouped cells.

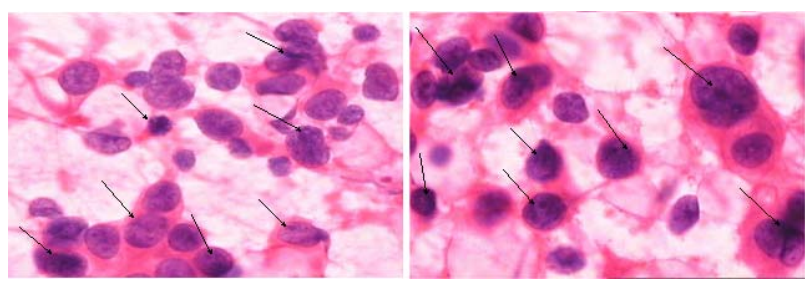

Fig. 2. Size, shape and staining variations as shown by the arrows: (a) G2 Ductal Carcinoma, (b) G3 Ductal Carcinoma.

All three factors are initialized to zero. According to the BR scheme, the malignancy of the tumor is assigned a grade that depends on the quantitative values of the above factors and is determined by the following equation:

$$
G=S D+P+H M F \text {. }
$$

The final grade is obtained by the summation of all the awarded points for each factor described earlier. Depending on the value of $G$, the tumor is assigned one of three grades:

- Grade I: Low malignancy.

- Grade II: Intermediate malignancy.

- Grade III: High malignancy.

These grades are determined according to the chart shown in Fig. 4

The evaluation of the malignancy of the tumor indicates the likelihood that the case can undergo metastasis at the time of or after the treatment. It also has an impact on the patient's type of treatment. Therefore, assigning a diagnosis to a case is a very difficult task and is dependent on the experience of the pathologist. More experienced pathologists that have seen more cases are more reliable in their diagnosis. On the other hand, due to overwork and fatigue, seeing more similar cases may lead to the misclassification of malignancy. To address this problem, we present an automated grading approach that is able to evaluate and assign a grade to fine needle aspiration biopsy tissue. To achieve this, we convert the Bloom-Richardson (Bloom and Richardson, 1957) grading scheme into a classification problem. In our method, the input FNA slice is first preprocessed and segmented 


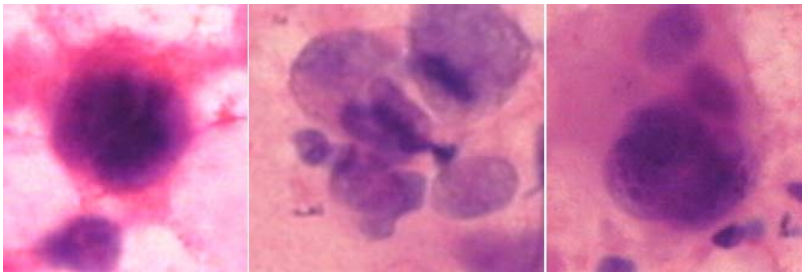

Fig. 3. Example of a mitosis.

according to the algorithm described in Section $2.1 \mathrm{Ba}-$ sed on the segmentation results, features taken into consideration are calculated (see Section 2.2) and used to train the classifier. Here we classify FNA slides into one of the three classes that represent a malignancy grade.

A detailed description of methods used in this study can be found in Section 2 followed by a comparison of the results obtained for the tested classifiers in Section 3 .

\section{Method}

Classification is a task of assigning an item to a certain category, called a class, based on the characteristic features of that item. This task in any classification system is performed by a classifier that takes a feature vector as an input and responds with a category to which the object belongs. A feature vector is a set of features extracted from the input data. Here we make use of neural network classifiers that are a collection of neurons (systems with many inputs and one output that are trained to fire, or not, for particular input patterns) that are connected one to another. Each connection is assigned an initial weight during the training process which are then adjusted to give a proper answer. The final decision is made based on the interaction of weights and the feature vector.

Before we can extract features used for classification, our input data need to be preprocessed and segmented. Preprocessing is a task of removing unimportant information from the data. Segmentation is an operation during which we isolate the boundaries of the important parts of data that are then used for feature extraction and classification.

The classification framework presented here consists of three main blocks: preprocessing, feature extraction and classification. Each of these blocks will be explained in detail in the following subsections.

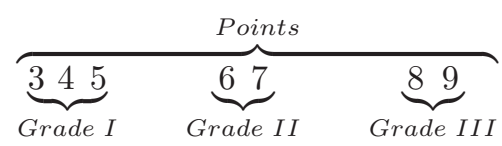

Fig. 4. Grade determination for the Bloom-Richardson grading scheme (Bloom and Richardson, 1957).
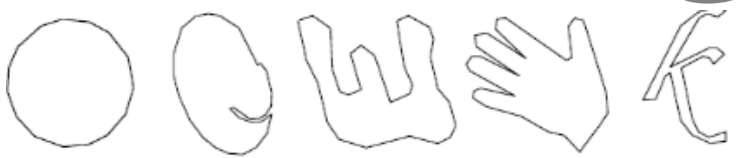

\section{$\begin{array}{lllll}1.0000 & 0.9681 & 0.6651 & 0.7702 & 0.3258\end{array}$}

Fig. 5. Graphical representation of convexity (Zunic and Rosin, 2002).

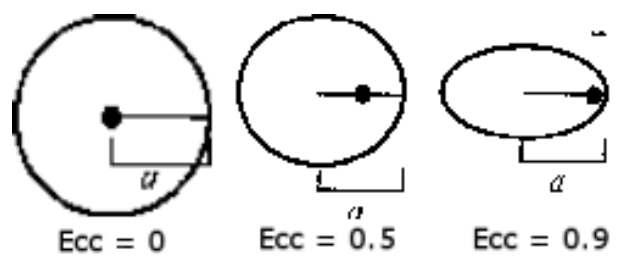

Fig. 6. Graphical representation of eccentricity.

2.1. Preprocessing. Preprocessing is a complicated process that has a tremendous impact on feature extraction and further on the classification error in any classification system. In this work we make use of the automated segmentation procedure that involves the level set method proposed by Li et al. (2005). Compared with other methods, such as the Hough transform (Ballard, 1981), level sets seem to be a better choice for segmentation because of their better time performance. In the literature we can find approaches based on the generalized Hough transform (Street, Wolberg and Mangasarian, 1993; Lee and Street, 2000) that showed good segmentation results due to the use of an elliptical shape to identify the location and size of nuclei. These approaches are computationally intensive and therefore time consuming (Jeleń, Krzyżak and Fevens, 2006). On the other hand, level set methods, which, also proved to be a powerful tool for medical image segmentation (Li, Xu, Gui and Fox, 2005; Droske, Meyer, Rumpf and K., 2001; Deng and Tsui, 2002; Tsai, Yezzi, Wells, Tempany, Tucker, Fan, Grimson and Willsky, 2003; Li, Fevens, Krzyżak, Jin and Li, 2006), involve fewer computations than Hough transform approaches and therefore achieve faster computational times.

Level sets were first described by Osher and Sethian (1988) as a method for capturing moving fronts. In the level set formulation, the segmentation problem is equivalent to the computation of a surface $\Gamma(t)$ that propagates in time along its normal direction. The $\Gamma$ surface is also called a propagating front, which, according to Osher and Sethian (1988), is embedded as a zero level of a time-varying higher dimensional function $\phi(x, t)$ :

$$
\Gamma(t)=\left\{x \in \mathbb{R}^{3} \mid \phi(x, t)=0\right\} .
$$

An evolution equation for an interface $\Gamma$, where $\Gamma$ is a closed curve in $\mathbb{R}^{2}$, can be written in a general form (Sethian 


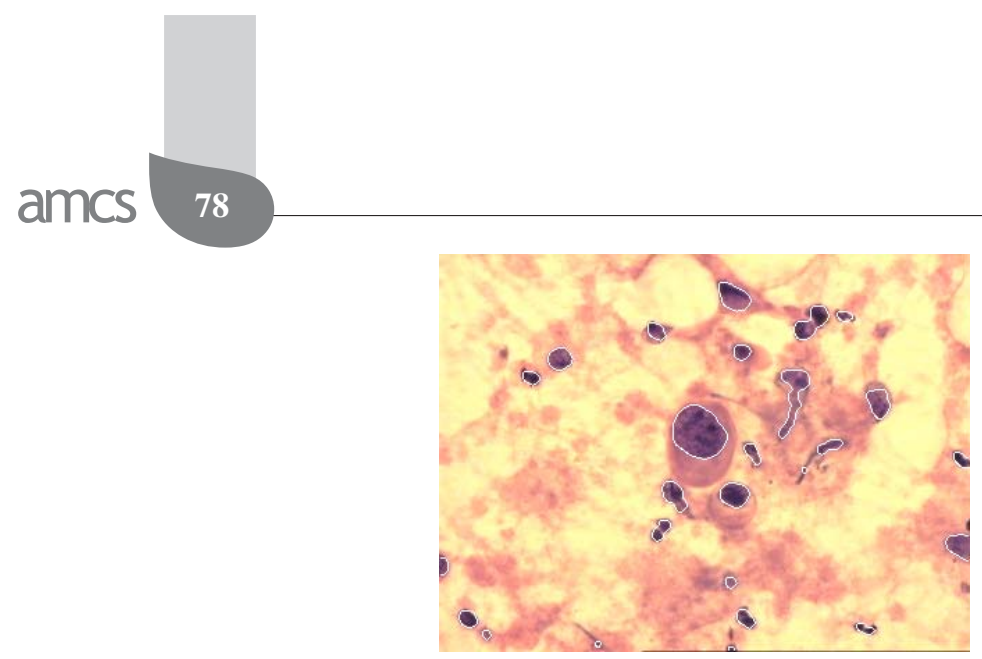

(a)

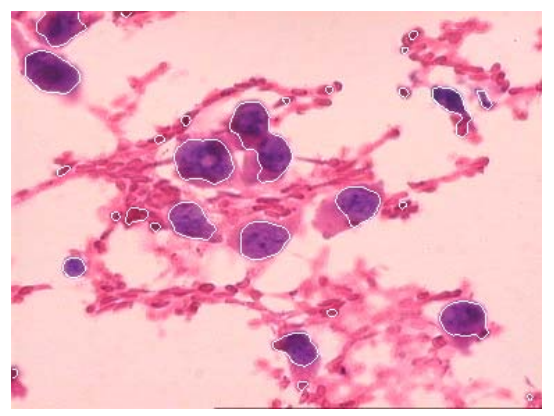

(c)

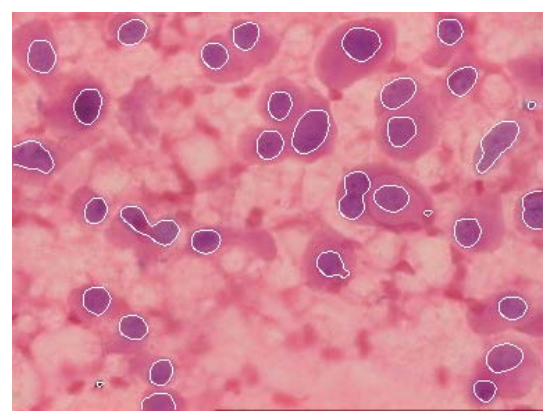

(e)

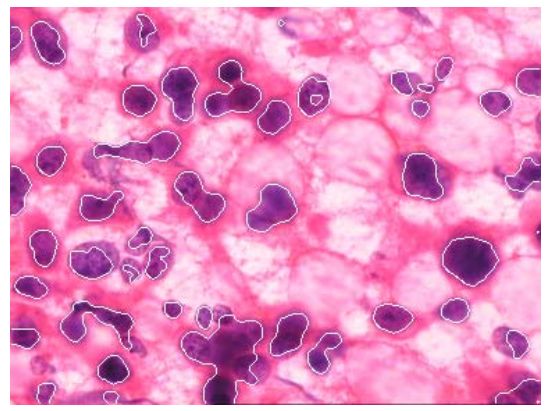

(b)

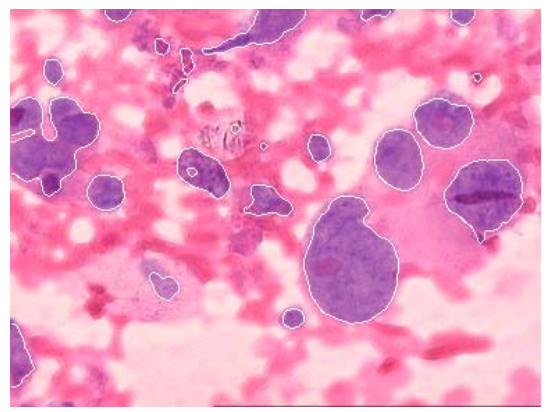

(d)

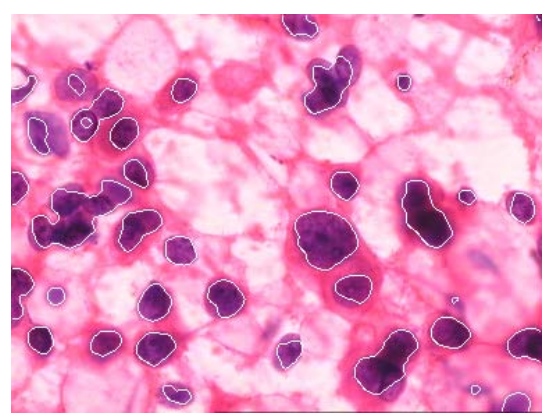

(f)

Fig. 7. Segmentation results: (a), (c), (d) and (e) represent intermediate malignancy, while (b) and (f) correspond to high malignancy.

and Adalsteinsson, 1997)

$$
\frac{\partial \phi}{\partial t}+F|\nabla \phi|=0
$$

The function $\phi$ describes a curve defined by $\phi(x, t)=d$, where $d$ is a signed distance between $x$ and the surface $\Gamma$. If $x$ is inside (resp. outside) of $\Gamma$, then $d$ is negative (resp. positive). The function $F$ is a scalar speed function that depends on image data and the function $\phi$.

The main drawback of this procedure is that during the evolution, $\phi$ can assume sharp or flat shapes. To overcome this problem, $\phi$ is initialized as a signed distance function before the evolution. Later, during the evolution, it is periodically reshaped to be a signed distance function (Li et al., 2005).

In our framework, we make use of variational level sets (Li et al., 2005), which are more robust than those originally proposed by Osher and Sethian because they 


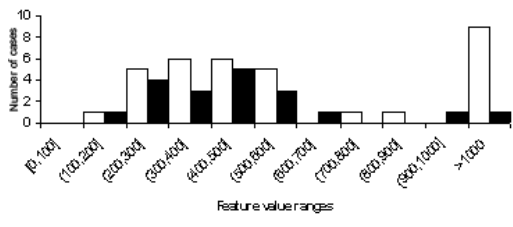

(a)

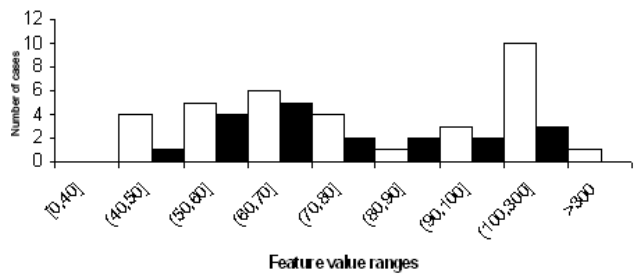

(b)

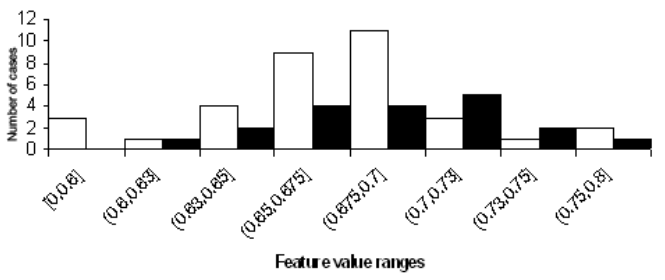

(c)

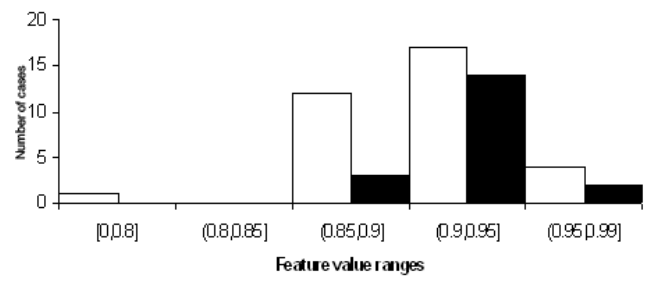

(d)

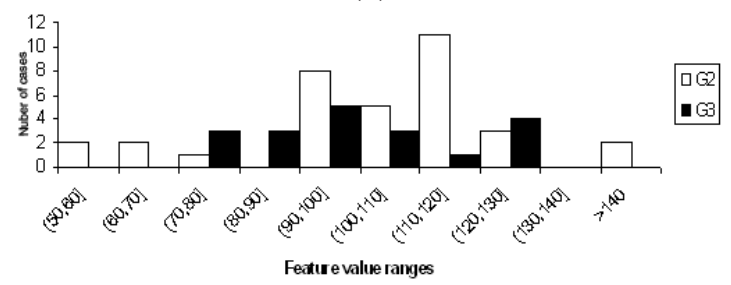

(e)

Fig. 8. Histograms of feature values for intermediate (G2) and high (G3) malignancy classes: (a) area, (b) perimeter, (c) eccentricity, (d) convexity and (e) texture.

All level set methods start with an initial level set function. The closer the initial level set function is to the final segmentation, typically, the more likely the level set method will quickly converge to the segmentation. Therefore, to automate the segmentation process and start with a good initial level set function, we make use of an iterative clustering approach for automatic image thresholding. This method was proposed by Riddler and Calvard (1978). In principle, their method seeks a threshold $T$, represented by a curve, within an image, which is restricted to have a bimodal histogram, and the final threshold level

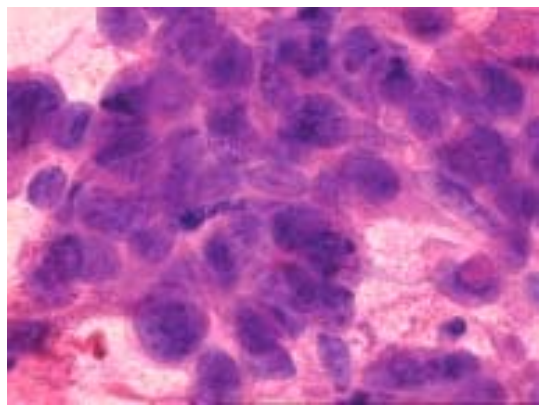

(a)

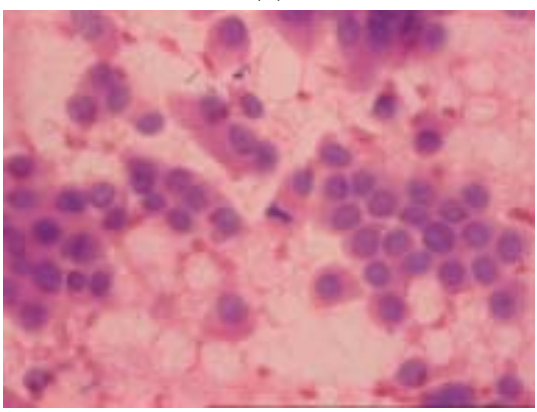

(b)

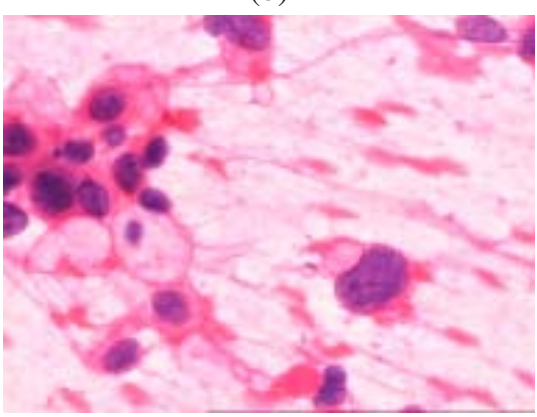

(c)

Fig. 9. Classification results: (a) intermediate malignancy misclassified as high, (b) intermediate malignancy correctly classified, (c) high malignancy correctly classified.

is calculated as

$$
T=\frac{\mu_{1}+\mu_{2}}{2},
$$

where $\mu_{1}$ and $\mu_{2}$ are the means of the components separated by $T$.

Due to the staining process of FNA images, the red channel provides best information about nuclear structures out of the three RGB channels. During the staining process nuclei stain with a shades purple and, when the red channel is extracted, all the nuclear features are preserved while the background information is lost. This observation allows us to extract and threshold the image red channel and then use it as an initial contour that is required by level sets. For our classification framework we make use of the level set proposition of (Li et al., 2005) with an initial level set obtained by thresholding with the Ridler 
and Calvard method (Ridler and Calvard, 1978). Some of the segmentation results are shown in Fig.7

2.2. Feature extraction. The features presented here were chosen to correspond to the indicators used for the Bloom-Richardson scheme. To precisely estimate the necessary features, only nuclear features, rather than cellular features, are taken into consideration because only these features are most discriminant and these are the features graded by pathologists. Taking this into consideration, as well, we use four shape-based features and one textural feature. The values obtained for these features yield a good differentiation between cancerous and healthy cells. For classification purposes we use the following features:

- Area: It is calculated as the sum of all pixels $(x)$ of the segmented nucleus $(N)$ :

$$
A=\sum_{x \in N} 1
$$

- Perimeter: It is the length of the nuclear envelope calculated as the length of a polygonal approximation of the boundary $(B)$ :

$$
p=\sum_{x \in B} 1
$$

- Convexity: It is calculated as the ratio of the nucleus area and its convex hull (Zunic and Rosin, 2002), which is the minimal area of the convex polygon that can contain the nucleus:

$$
C(S)=\frac{A}{\operatorname{Area}(C H(S))},
$$

where $S$ is a nucleus and $C H(S)$ is its convex hull. Convex shapes will yield a value of 1 , while concave shapes will have a value less than 1 (see Fig. 5).

- Eccentricity: It allows us to track how much a segmented nucleus differs from a healthy nucleus. Healthy nuclei will assume circular shapes while cancerous nuclei can assume arbitrary shapes. We calculate eccentricity as the ratio of the distance between the foci of an ellipse, which has the same second moments as the extracted nuclei, and the length of its major axis. The values of this feature vary between 0 and 1. These are degenerate cases because a shape whose eccentricity is 0 is actually a circle, while a shape whose eccentricity is 1 is a line segment (see Fig. 6).

- Texture measure: It represents nuclear intensity changes in the image. We calculate this feature as an average red channel value of a nucleus.
The first four features describe changes in the size and shape of the cancer nuclei. The texture measure describes changes in the staining of the nuclei and is also used as a feature for the third BR factor, i.e., the frequency of hyperchromatic and mitotic figures.

The classification stage of the system that uses these features is described in the following subsection and the obtained results are presented in Section 3

2.3. Classification. Here we make use of neural networks based on support vector machines for classification purposes. The classification process is based on transforming a feature vector into a higher-dimensional space where a separating hyperplane is constructed. During the training process only those vectors that are closest to the separating plane are used because they carry the most valuable information about classification. The SVMs used in this study use the idea of large margin classifiers for training that provides a good generalization of the problem. Large margin classifiers use kernel-based methods for data separation.

The learning process uses the Adatron algorithm (Friess, Cristianini and Campbell, 1998) that was extended by the substitution of the inner product of patterns in the input space by the kernel function which yields

$$
J(\alpha)=\sum_{i=1}^{N} \alpha_{i}-\frac{1}{2} \sum_{i=1}^{N} \sum_{j=1}^{N} \alpha_{i} \alpha_{j} d_{i} d_{j} G_{2 \sigma^{2}}\left(x_{i}-x_{j}\right),
$$

with the following constrains:

$$
\sum_{i=1}^{N} d_{i} \alpha_{i}=0 \quad \alpha_{i} \geq 0, \quad i=1, \ldots, N,
$$

where $x_{i}, x_{j}$ are feature vectors, $d_{i}, d_{j} \in\{2,3\}$ are malignancy grades, $\alpha_{i}$ are multipliers and $G$ is a Gaussian kernel with variance $\sigma$. We also have

$$
\begin{gathered}
g(x)=d_{i}\left(\sum_{j=1}^{N} d_{j} \alpha_{j} G_{2 \sigma^{2}}\left(x-x_{j}\right)+b\right), \\
\alpha_{i}(n+1)= \\
\begin{cases}\alpha_{i}(n)+\Delta \alpha_{i}(n) & \text { if } \alpha_{i}(n)+\Delta \alpha_{i}>0, \\
\alpha_{i}(n) & \text { if } \alpha_{i}(n)+\Delta \alpha_{i} \leq 0, \\
M= & \min _{i} g(x),\end{cases}
\end{gathered}
$$

where $g$ is a decision boundary, $\alpha_{j}$ is nonzero if and only if $x_{j}$ is a support vector.

Training starts with a starting multiplier $\alpha_{i}=0.1$ and a terminating threshold $t=0.01$. We calculate $\Delta \alpha_{i}=$ $\eta[1-M]$ and perform an update according to Eqn. 12] as long as $M>t, \eta$ is a predefined learning rate. 
Table 1. Some of the calculated features along with pathologist grading.

\begin{tabular}{|c|c|c|c|c|c|c|}
\hline Feature & Case 1 & Case 2 & Case 3 & Case 4 & Case 5 & Case 6 \\
\hline \hline Area & 591 & 434 & 617 & 184 & 218 & 320 \\
Perimeter & 80.5 & 70.0 & 105.0 & 40.8 & 48.0 & 56.2 \\
Convexity & 0.9420 & 0.9454 & 0.9045 & 0.9420 & 0.9561 & 0.9640 \\
Eccentricity & 0.7180 & 0.6761 & 0.6547 & 0.6920 & 0.6036 & 0.6000 \\
Texture & 116.7 & 124.7 & 120.3 & 98.0 & 123.8 & 108.2 \\
\hline BR Grade & G3 & G3 & G3 & G2 & G2 & G2 \\
\hline
\end{tabular}

Table 2. Training and testing sets used for classification.

\begin{tabular}{|c|c|c|c|c|}
\hline \multirow{2}{*}{} & \multicolumn{2}{|c|}{ G2 } & \multicolumn{2}{c|}{ G3 } \\
\cline { 2 - 5 } & Training [\%] & Testing [\%] & Training [\%] & Testing [\%] \\
\hline set \#1 & 50 & 50 & 30 & 70 \\
\hline set \#2 & 50 & 50 & 50 & 50 \\
\hline set \#3 & 50 & 50 & 70 & 30 \\
\hline set \#4 & 50 & 50 & 90 & 10 \\
\hline set \#5 & 30 & 70 & 50 & 50 \\
\hline set \#6 & 70 & 30 & 50 & 50 \\
\hline
\end{tabular}

Table 3. Error rates of the tested classifiers versus different training sets.

\begin{tabular}{|c|c|c|c|c|c|c|c|}
\hline Classifier & Set \#1 & Set \#2 & Set \#3 & Set \#4 & Set \#5 & Set \#6 & Average performance. \\
\hline SOM & $23.28 \%$ & $21.91 \%$ & $19.49 \%$ & $22.43 \%$ & $6.32 \%$ & $23.75 \%$ & $19.53 \%$ \\
MLP & $23.18 \%$ & $16.47 \%$ & $22.76 \%$ & $12.44 \%$ & $6.73 \%$ & $24.71 \%$ & $17.30 \%$ \\
SVM & $21.55 \%$ & $20.25 \%$ & $20.91 \%$ & $23.15 \%$ & $5.76 \%$ & $24.71 \%$ & $19.39 \%$ \\
PCA & $22.84 \%$ & $16.32 \%$ & $20.88 \%$ & $22.01 \%$ & $7.22 \%$ & $23.66 \%$ & $18.82 \%$ \\
\hline
\end{tabular}

To classify FNA cytological tissue, we use an SVM classifier that takes a five-element feature vector as an input and responds with a two-element output vector $(1,0)^{T}$ for intermediate malignancy and $(0,1)^{T}$ for high malignancy.

To compare the performance of the SVM classifier, we make use of additional tree classifiers such as the Multilayer Perceptron (MLP), Self-Organizing Maps (SOM), Principal Components-based neural networks (PCA). MLPs are simple feedforward neural networks trained with the backpropagation method in a supervised manner (Duda, Hart and Stork, 2000). SOM neural networks reduce the input space into representative features according to a self-organizing process (Kohonen, 1990) and are trained in an unsupervised manner. PCA neural networks are a combination of supervised and unsupervised trained neural networks. PCA analysis finds solutions in an unsupervised manner from input data and then supervised MLPs are used for the classification of the components (Oja, 1982).

\section{Results}

In this section we will demonstrate the performance of the framework presented in Section 2 along with results obtained for three other classifiers for comparison. The classifiers were trained and tested with images from our database of FNA slides. All of the images were stained with the Haematoxylin and Eosin techniques (HE), which yielded purple and black stains for nuclei, shades of pink for cytoplasm and orange/red for red blood cells. All the images were obtained with an Olympus BX 50 microscope with a mounted CCD-IRIS camera connected to a PC computer with the MultiScan Base 08.98 software.

Our database consists of 110 fine needle aspiration biopsy images with known malignancy grades collected at the Department of Pathology at the Medical University of Wrocław. There are 44 images with high malignancy (G3) and 66 images with intermediate malignancy (G2). Benchmark grades of the images were assigned by a pathologist and used for verification purposes during the te- 
sts. Since 2005 none of the performed biopsies has been assigned a low malignancy grade, therefore all cases included in the database are graded as intermediate or high malignancy. Figure 7 shows an example of segmentation that was used for feature extraction and Table 1 shows some of the extracted features along with malignancy grades assigned by an expert pathologist. Figure 8 shows histograms of features for both classes. From the table we can see that features extracted by our framework reflect the tendency of malignant cancer cells, where more malignant cases have larger nuclei and their border is more deformed than those of healthy nuclei. Using such feature vectors, we performed six different tests on our database with different training and testing sets.

The training sets were chosen randomly to contain different numbers of high and intermediate malignancy cases. The remaining cases were used to form the test sets. Table 2 shows the chosen training and testing sets that were used in this study. Using these sets we trained and tested the performance of four different classifiers. We took into consideration four neural networks: a Multilayer Perceptron (MLP), Self-Organizing Maps (SOM), Principal Components (PCA) and Support Vector Machines (SVM), cf. Section 2.3, and compared their performance using our database.

Out of the four classifiers, the MLP showed the best average performance with an average error rate of $17.3 \%$. Although the MLP showed the best average performance, we can conclude that SVMs perform better achieving an error rate of $5.76 \%$, which is the lowest recorded error rate. The highest recorded error rate for the test set \#5 was $7.22 \%$ for the PCA. For the same training and testing sets, the MLP performed significantly worse than the SVM, achieving a $6.73 \%$ error rate.

Table 3 and Fig. 10 show the performance of all classifiers for each set taken into consideration in this study. From Fig. 10 one can see that the set $\# 5$ achieved the best performance, having the lowest error rates out of all testing sets taken into consideration. In pattern recognition and machine learning, Receiver Operating Characteristics (ROC) are widely used for performance analysis and provide tools to select a possibly optimal classification model. An ROC shows that the SVM performed better that any other tested classifier. When comparing the area under the ROC curve (AUC), one can notice that for the SVM the area is larger than for any other classifier taken into consideration in this study. According to ROC analysis, we choose the classifier with the largest AUC (Bradley, 1997). This is reflected in Fig. 11, which shows comparative performance of all tested classifiers.

In Fig. 9 we present examples of the misclassified case (Fig. 9.a)) and two correctly classified cases (Figs. 9 (b) and (c)).

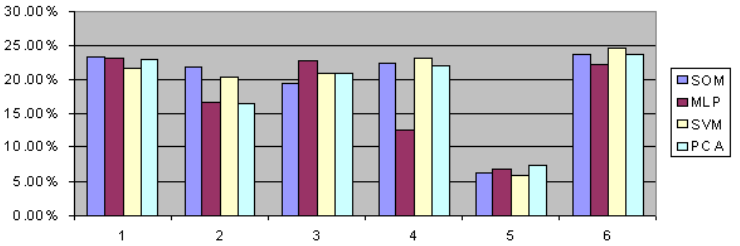

Fig. 10. Error rates of the tested classifiers for each training set.

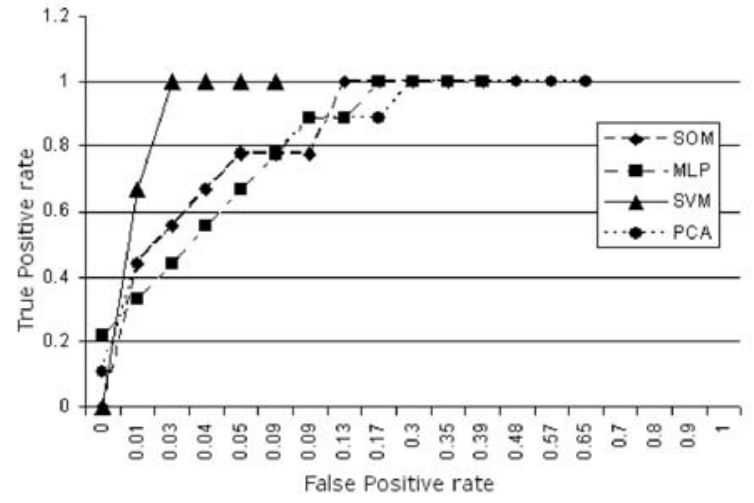

Fig. 11. ROC curves for all classifiers

\section{Conclusions}

In Section 2 we presented a framework for breast cancer malignancy grading based on an SVM classifier that was able to classify malignancy and, based on that classification, assign a malignancy grade to fine needle aspiration biopsy slides.

In Section 2.1 the preprocessing part of the system was described. From the previous work (Jeleń et al., 2006), where the Hough transform approach to nuclei classification was described, we can conclude that the level set approach is a faster method for nuclei segmentation and also provides a good nuclei representation (see Fig. 77.

The performance of our system given in Section 3 shows that SVMs are able to classify malignancy with very good results, achieving the highest accuracy of 94.24\% for the training set \#5, which includes only $30 \%$ of $\mathrm{G} 2$ cases and $50 \%$ of G3 cases. Using only $30 \%$ of G2 cases introduces less redundant information, which showed a very good influence on classification results.

The $5.76 \%$ error rate is very promising and allows us to further investigate this problem on feature extraction and preprocessing levels to achieve better classification rates. Another possibility to lower the error would be to increase the database to include more malignancy cases, which we are currently investigating. From a pathologist point of view, having a system that is able to assist them during the decision making process is very useful and helpful especially in situations where it is difficult to 
decide to which malignancy class a case should belong. Such a system would help to make their diagnosis more objective and precise.

\section{Acknowledgements}

We are grateful for the support of Professor Michał Jeleń from the Department of Anatomical Pathology at the Medical University of Wrocław, who provided us with images that were used in this study.

\section{References}

Ballard H. (1981). Generalizing the Hough transform to detect arbitrary Shapes, Pattern Recognition 13(2): 111-122.

Bloom H. and Richardson W. (1957). Histological grading and prognosis in breast cancer, British Journal of Cancer, 11(3): 359-377.

Bradley A. (1997). The use of the area under the ROC curve in the evaluation of machine learning algorithms, Pattern Recognition 30(7): 1145-1159.

Deng J. and Tsui H. (2002). A fast level set method for segmentation of low contrast noisy biomedical images, Pattern Recognition Letters 23(1-3): 161-169.

Droske M., Meyer B., Rumpf M. and K. S. (2001). An adaptive level set method for medical image segmentation, Lecture Notes in Computer Science 2082: 416-422.

Duda R., Hart P. and Stork D. (2000). Pattern Classification, 2nd edn, Wiley Interscience Publishers, New York.

Friess T., Cristianini N. and Campbell C. (1998). The kernel adatron algorithm: A fast and simple learning procedure for support vector machines, Proceedings the 15th International Conference on Machine Learning, Morgan Kaufman Publishers, San Francisco, USA, pp. 188-196.

Jeleń Ł., Krzyżak A. and Fevens T. (2006). Automated feature extraction for breast cancer grading with BloomRichardson scheme, International Journal of Computer Assisted Radiology and Surgery 1(1): 468-469.

Kohonen T. (1990). The self-organizing map, Proceedings of Institute of Electrical and Electronics Engineers, 78(9): 1464-1480.

Lee K. and Street W. (2000). Generalized Hough transforms with flexible templates, Proceedings of International Conference on Artificial Inteligence, Las Vegas, NV, pp. 11331139.

Li C., Xu C., Gui C. and Fox M. (2005). Level set evolution without re-initialization: A new variational formulation, Proceedings of the IEEE Conference on Computer Vision and Pattern Recognition, CVPR 2005, San Diego, USA, pp. 430-436.

Li S., Fevens T., Krzyżak A., Jin C. and Li S. (2006). Fast and robust clinical triple-region image segmentation using one level set function, Proceedings of the International Conference on Medical Image Computing and Computer Assisted Intervention, Copenhagen, Denmark, 2: pp. 766-773.
Nezafat R., Tabesh A., Akhavan S., Lucas C. and Zia M. (1998). Feature selection and classification for diagnosing breast cancer, Proceedings of International Association of Science and Technology for Development International Conference, Cancun, Mexico, pp. 310-313.

Oja E. (1982). A siplified neuron modeled as a principal component analyzer, Journal of Mathematical Biology 15(3): 267-273.

Osher S. and Sethian J. (1988). Fronts propagating with curvature-dependent speed: Algorithms based on Hamilton-Jacobi formulations, Journal of Computational Physics 79: 12-49.

Ridler T. and Calvard S. (1978). Picture thresholding using an iterative selection, IEEE Transactions on System, Man and Cybernetics 8: 630-632.

Schnorrenberg F., Pattichis C., Kyriacou K. and Schizas C. (1994). Detection of cell nuclei in breast cancer biopsies using receptive fields, IEEE Proceedings of Engineering in Medicine and Biology Society, pp. 649-650.

Sethian J. and Adalsteinsson D. (1997). An overview of level set methods for etching, deposition and lithography development, IEEE Transactions on Semiconductor Manufacturing 10(1): 167-184.

Street N. (2000). Xcyt: A system for remote cytological diagnosis and prognosis of breast cancer, in L. Jain (Ed.), Soft Computing Techniques in Breast Cancer Prognosis and Diagnosis, World Scientific Publishing, Singapore, pp. 297-322.

Street W. N., Wolberg W. H. and Mangasarian O. L. (1993). Nuclear feature extraction for breast tumor diagnosis, Proceedings of the International Symposium on Electronic Imaging: Science and Technology, Vol. 1905, San Jose, CA, USA, pp. 861-870.

Tsai A., Yezzi A., Wells III W., Tempany C., Tucker D., Fan A., Grimson W. and Willsky A. (2003). A shape-based approach to the segmentation of medical imagery using level sets, Medical Imaging 22(2): 137-154.

Walker H. J. and Albertelli L. (1998). Breast cancer screening using evolved neural networks, Proceedings of the IEEE International Conference on Systems, Man and Cybernetics, San Diego, USA, pp. 1619-1624.

Walker H. J., Albertelli L., Titkov Y., Kaltsatis P. and Seburyano G. (1998). Evolution of neural networks for the detection of breast cancer, Proceedings of International Joint Symposia on Inteligence and Systems pp. 34-40.

Wolberg W. H., Street W. N. and Mangasarian O. L. (1994). Machine learning techniques to diagnose breast cancer from image-processed nuclear features of fine needle aspirates, Cancer Letters 77: 163-171.

Wolberg W. and Mangasarian O. (1990). Multisurface method of pattern separation for medical diagnosis applied to breast cytology, Proceedings of the National Academy of Science, USA 87(23): 9193-9196.

Zunic J., and Rosin P. (2002). A convexity measurement for polygons., Proceedings of the British Machine Vision Conference Cardiff, UK, 24: 173-182. 
